\title{
The dynamics of self-care in the course of heart failure management: data from the INTOUCH study
}

This article was published in the following Dove Press journal:

Patient Preference and Adherence

\begin{abstract}
Edita Lycholip, ${ }^{1,2}$ Ina Thon
Aamodt, ${ }^{3,4}$ Irene Lie, ${ }^{3}$

Toma Šimbelytė, ${ }^{5}$ Roma

Puronaitè, ${ }^{2,6,7}$ Hans Hillege, ${ }^{8}$

Arjen de Vries, ${ }^{8}$ Imke Kraai, ${ }^{9}$

Anna Stromberg, ${ }^{10}$ Tiny

Jaarsma, " Jelena Čelutkienè ${ }^{1,2}$

'Institute of Clinical Medicine, Faculty of Medicine, Vilnius University, Vilnius, Lithuania; ${ }^{2}$ Center of Cardiology and Angiology, Vilnius University Hospital Santaros Clinics, Vilnius, Lithuania; ${ }^{3}$ Centre for Patient-Centered Heart and Lung Research, Department of Cardiothoracic Surgery, Division of Cardiovascular and Pulmonary Diseases, Oslo University Hospital, Oslo, Norway; ${ }^{4}$ Department of Nursing Science, Institute of Health and Society, Faculty of Medicine, University of Oslo, Oslo, Norway; ${ }^{5}$ Clinic of Internal Medicine, Centre of Family and Internal Medicine, Vilnius University Santaros Clinics, Vilnius University, Vilnius, Lithuania; ${ }^{6}$ Centre of Informatics and Development, Vilnius University Hospital Santaros Clinics, Vilnius, Lithuania; ${ }^{7}$ Institute of Mathematics and Informatics, Vilnius University, Vilnius, Lithuania; ${ }^{8}$ Department of Cardiology, University Medical Center Groningen, University of Groningen, Groningen, the Netherlands; ' PRA Health Sciences - Early Development Services, Groningen, the Netherlands; ${ }^{10}$ Department of Medical and Health Sciences, Linkoping University, Linkoping, Sweden; "Department of Socia and Welfare Studies, Linkoping University, Norrkoping, Sweden
\end{abstract}

Correspondence: Edita Lycholip Center of Cardiology and Angiology, Vilnius University Hospital Santaros Clinics, Santariškių 2, Vilnius 0860I, Lithuania Tel +370650817 72 Email edita.lycholip@santa.lt
Introduction: Self-care is an important patient-reported outcome (PRO) for heart failure (HF) patients, which might be affected by disease management and/or telemonitoring (TM). The number of studies reporting the influence of TM on self-care is limited.

Aims: This study aimed: to assess whether TM, in addition to information-and-communicationtechnology (ICT)-guided disease management system (ICT-guided DMS), affects self-care behavior; to evaluate the dynamics of self-care during the study; to investigate factors contributing to self-care changes; and to identify a patient profile that predisposes the patient to improvement in self-care.

Methods: In the INnovative ICT-guided-DMS combined with Telemonitoring in OUtpatient clinics for Chronic HF patients (IN TOUCH) study, 177 patients were randomized to either ICT-guided DMS or TM+ICT-guided DMS, with a follow-up of 9 months. The current analysis included 118 participants (mean age: $69 \pm 11.5$ years; $70 \%$ male) who filled the following PRO instruments: the nine-item European Heart Failure Self-care Behaviour scale (EHFScBs), Hospital Anxiety and Depression scale (HADs), and Minnesota Living with HF Questionnaire (MLHFQ).

Results: The baseline level of self-care was better in the TM+ICT-guided-DMS group $(\mathrm{n}=58)$ compared to ICT-guided-DMS group $(\mathrm{n}=60, p=0.023)$. Self-care behavior improved in the ICT-guided-DMS group $(p<0.01)$ but not in the TM+ICT-guided-DMS group. Factors associated with self-care worsening were as follows: higher physical subscale of MLHFQ (per 10 points, $p<0.05$ ), lower left ventricular ejection fraction (LVEF) (per 5\%, $p<0.05$ ), lower New York Heart Association (NYHA) class (class III vs class II, $p<0.05$ ). The subgroups of patients who had an initial EHFScBs total score $>28$, or from 17 to 28 with concomitant HADs depression subscale (HADs_D) score $\leq 8$, demonstrated the greatest potential to improve self-care during the study.

Conclusion: TM did not have an advantage on self-care improvement. Poor physical aspect of quality of life, lower LVEF, and lower NYHA class were associated with self-care worsening. The greatest self-care improvement may be achieved in those patients who have low or medium initial self-care level in the absence of depression.

Keywords: heart failure, self-care, telemonitoring, disease management, patient-reported outcomes

\section{Plain language summary}

Patient self-care is a key component of daily heart failure (HF) management and requires a thorough examination, because patients continue to report lack of understanding and participation. In this study, we not only looked at the self-care of the patients who were subjected to computed disease management and/or telemonitoring (TM) systems but also sought to clarify 
the factors associated with worsening or improving of self-care. We analyzed the data of the INnovative ICT-guided-DMS combined with Telemonitoring in OUtpatient clinics for Chronic HF patients (IN TOUCH) study and the patient-reported outcomes, using selfcare behavior, the Hospital Anxiety and Depression scale (HADs), and quality-of-life questionnaires. A very important part of the study is related to the identification of a patient profile that has the highest potential to change self-care behavior. Innovative nonparametric statistical methods, such as the regression tree, helped us to find that the dynamics of self-care depends on its initial level and depression. The physical aspect of quality of life, imaging parameters, and the functional class also were relevant characteristics. This study revealed that depression plays a major role in the change in self-care behavior in chronic HF patients and that it can worsen self-care by $>10$ times in a particular group of patients. This makes it possible to assume that in order to improve self-care behavior, dedicated programs should be adopted for patients with depressive symptoms and/or depressive symptoms should be treated first or in parallel with self-care-improving interventions.

\section{Introduction}

Self-care is an essential component of chronic heart failure (HF) management, necessary for improvement of the patient's well-being, quality of life, and prognosis. ${ }^{1}$ Self-care is a process of maintaining health through health-promoting practices and by managing illness, eg, by exercising, weight monitoring, taking medication, and seeking a health care provider when symptoms are deteriorating. ${ }^{1}$ A growing body of evidence supports the association between self-care and clinical outcomes such as event-free survival and reduced number of hospital admissions. ${ }^{2,3}$ Recently, a published metaanalysis of 20 randomized controlled trials (RCTs) confirmed that improvement of patients' HF self-care was related to decreased mortality and hospitalization rates. ${ }^{4,5}$ However, it is known that self-care is suboptimal in HF patients worldwide and cannot always be easily improved. ${ }^{6}$

While trying to design more effective interventions to improve HF management, it is important to know which factors determine self-care. A systematic review ${ }^{7}$ including 30 studies described several patient-dependent factors, some of them being consistently (depression) or inconsistently (age, gender, education, and left ventricular ejection fraction [LVEF]) associated with HF self-care behavior. No relationship was found between New York Heart Association (NYHA) class, health-related quality of life (HR-QoL), and HF self-care in that review. ${ }^{7}$ Other factors, reported in the literature as negatively affecting self-care in HF patients, are cognitive impairment, diabetes mellitus, and anxiety. ${ }^{8-13}$

Several interventions have been tested to improve selfcare in HF patients, such as goal setting, using diaries, educational programs, or social support. ${ }^{4}$ A new way to improve outcomes in chronic diseases is the use of homebased TM, which allows the direct transmission of patientrelated data from home to health care professionals. Several RCTs demonstrate a positive impact of noninvasive daily TM on outcomes such as the number of days lost to hospitalization, HF-related hospitalizations, all-cause mortality, HF knowledge, and self-care. ${ }^{14-17}$ However, the number of RCTs reporting changes of self-care while using TM is limited, and some studies found no improvement in this PRO comparing TM and standard management groups. ${ }^{17,18}$ The data for the present study were collected during a larger multicenter RCT, the INnovative ICT-guided-DMS combined with Telemonitoring in OUtpatient clinics for Chronic HF patients (IN TOUCH) study, investigating TM, in addition to the information-and-communication-technology (ICT)guided disease management system (ICT-guided DMS). ${ }^{19}$

\section{Aims of the study}

The study had the following aims:

1. to assess whether TM plus ICT-guided DMS has an effect on self-care;

2. to evaluate the general dynamics of HF self-care in the IN TOUCH study population;

3. to describe the factors contributing to the deterioration or improvement of self-care; and

4. to identify which patient characteristics are associated with the highest potential to change self-care behavior.

\section{Methods}

\section{Trial design}

The methods and design of the IN TOUCH study were reported elsewhere, ${ }^{19,20}$ but are shortly described here. Briefly, IN TOUCH was a multicenter RCT conducted in ten Dutch hospitals. This study consisted of two intervention groups: ICT-guided DMS with and without TM. The primary end point (a composite weighted score consisting of a value each for mortality, HF readmission, and change in quality of life measured with the Minnesota Living with HF Questionnaire [MLHFQ]) and most secondary end points (the total number and duration of all hospital admissions, as well as cost analyses) did not significantly differ between the two study groups. The only documented effect of TM was a reduction in visits to the HF outpatient clinic $(p<0.02) .{ }^{19}$

Patients were randomized within 2 weeks after discharge or at their first outpatient clinic visit due to HF deterioration and were followed for 9 months.

The medical ethical committee of the Medical University of Groningen approved the protocol of the IN TOUCH study 
in the Netherlands (approval number ABR:NL26271.042.08). All participants provided written informed consent.

\section{Data collection}

\section{Clinical data}

NYHA class determination, physical examination, electrocardiogram (ECG) recordings, and laboratory tests were completed at baseline, 2 weeks, and 9 months, while echocardiography was conducted only at baseline. Patient-reported outcomes (PROs), including self-care, depression and anxiety symptoms, and HR-QoL, were evaluated at baseline and at the end of the study.

\section{Self-care}

The European Heart Failure Self-care Behaviour scale $(\mathrm{EHFScBs})^{21}$ is a questionnaire of nine items about a patient's actions related to HF self-care (nonpharmacological and pharmacological treatment adherence, observation of HF symptoms and signs, and measures that the patient takes when his/her condition starts to deteriorate). Each answer is graded from one (totally agree) to five (totally disagree), with higher scores reflecting poorer self-care. The changes in the EHFScBs scores were compared between the groups and in the whole study population during the study. Self-care was considered to worsen or improve if the total EHFScBs score increased or decreased by at least one point, respectively, and not to change if the difference in scores was equal to zero.

\section{Anxiety and depression}

The HADs consists of 14 questions, and each has four choices rated from zero (not present) to three (maximum) points. Seven questions evaluate depressive symptoms - depression subscale (HADs_D), and the other seven reveal anxiety symptoms - anxiety subscale (HADs_A). Scores range from 0 to 21 in each of the two (depression and anxiety) parts and demonstrate the severity of depression or anxiety. Scores from zero to seven are interpreted as the absence of anxiety or normal mood; from eight to ten - as mild; from 11 to 14 - as moderate; and from 15 to 21 - as severe anxiety or depression. ${ }^{22,23}$

\section{HR-QoL assessment}

The MLHFQ ${ }^{24,25}$ is a 21 -item disease-specific HR-QoL instrument, with scores for each item asking how much the HF prevented from living as wanted during the previous month. The response options range from "no" (score: 0), "very little" (score: 1) to "very much" (score: 5). The total score ranges between zero and 105, the physical dimension (subscale) between 0 and 40, and the emotional dimension (subscale) between zero and 25. Higher scores represent worse HR-QoL.

\section{Patient population}

The inclusion criteria were as follows: admission to the intensive care/coronary care unit or cardiology ward or visiting the outpatient HF clinic and in need of treatment or adjustment with oral or intravenous diuretics, aged 18 years or older, evidence of structural underlying heart disease, documented reduced LVEF $\leq 45 \%$. Exclusion criteria were myocardial infarction in the past month, cardiac invasive intervention in the past 6 months or planned in the next 3 months, hemodialysis, use of other TM systems, and inability or unwillingness to give informed consent. ${ }^{19}$ Overall, 177 patients were enrolled in the study from December 2009 to January 2012; 83 patients were randomized to the ICT-guided-DMS group and 94 patients to TM+ICT-guided-DMS group. The current analysis included 118 participants who filled the PRO questionnaires mentioned earlier.

\section{Study interventions}

Patients in the ICT-guided-DMS group were evaluated and educated at the HF clinic by a HF nurse according to existing European Society of Cardiology (ESC) guidelines. ${ }^{26}$ Medical history, physical examination results, nursing assessment, laboratory test results, and questionnaire information were loaded into the ICT-guided-DMS and used to give some advice on medication uptitration to the health care providers during the clinic visit.

The TM+ICT-guided-DMS group had additional devices at home to measure body weight and blood pressure, as well as to record an ECG twice a week when increasing the betablocker dose. The measurements obtained from additional devices were directly transmitted into the DM system; in case the measured values were out of range, an interactive monitor generated the questions regarding the patient's condition and advice about the nonpharmacological treatment. Then, the HF nurse automatically received notification by mobile phone and e-mail and, within 2 hours, discussed the symptoms and treatment with the patient. Furthermore, the patients in the TM group could visit the clinic or hospital only if there was a definite need for intervention.

\section{Statistical analysis}

First, within-group and between-group comparisons were made using Mann-Whitney and chi-square test for baseline characteristics and PROs; paired Student's $t$-test and Wilcoxon signed-rank test were used to evaluate changes in self-care over time in the total group and in both study groups 
separately. To assess HF severity, an additional analysis was performed to compare the levels of NT-pro-BNP between the 118 patients included in the present study on the one hand and the whole IN TOUCH population on the other: the data were divided into deciles; using the chi-square criterion (goodness of fit), we verified the distribution of the analyzed sample according to previously received intervals.

Second, a logistic regression analysis was performed to determine the association of the study covariates with improvement, no change, or worsening in self-care. The independent variables included the intervention group, age, gender, the MLHFQ score, the NYHA functional class, the level of $N$-terminal pro-brain natriuretic peptide (NT-pro-BNP), LVEF, and the HADs_D and HADs_A scores. Variables that were significant in the univariate analysis were included into the multivariate stepwise logistic regression.

Third, to identify subgroups with the greatest potential to improve self-care, conditional inference trees were constructed. This is a nonparametric method that is selfexplanatory and easily interpretable by nonprofessional users in classification tasks. ${ }^{27}$ In this case, a regression tree - with a continuous target variable of change in self-care and with input variables similar as in the logistic regression analysis was used. Finally, based on the regression tree distribution, the impact of depression factor was separately investigated by univariate regression analysis in three groups with different initial self-care levels.

The Statistical Package for Social Sciences (SPSS), version 20 (released 2011, IBM Corp, Armonk, NY, USA) and R package "party" were used for statistical analysis. Moreover, $p$-values $<0.05$ were considered significant.

\section{Results}

The current analysis included 118 participants who completed PROs; of these, 60 and 58 patients were randomized into the ICT-guided-DMS and the TM+ICT-guided-DMS groups, respectively. Compared to the 177 patients in the original study, the 118 patients were of similar age with less males in the TM group (64\% vs $70 \%$, respectively); the NT-pro-BNP level was not significantly different from that of patients in the whole study $(p=0.749)$.

\section{Patient characteristics}

The mean age of the patients was $69 \pm 11.5$ years, $70 \%$ were male, $81 \%$ were classified as NYHA class III/IV, and mean LVEF was $27 \% \pm 9.9 \%$. The baseline patient characteristics are presented in Table 1.
Table I Baseline patient characteristics

\begin{tabular}{|c|c|c|c|}
\hline Characteristics & $\begin{array}{l}\text { ICT-guided } \\
\text { DMS; } n=60\end{array}$ & $\begin{array}{l}\text { TM+ICT-guided } \\
\text { DMS; } n=58\end{array}$ & $p$-value \\
\hline \multicolumn{4}{|l|}{ Demographics } \\
\hline Age (years) & $69.5 \pm 10.4$ & $68.5 \pm 12.7$ & 0.94 \\
\hline Male gender & $46(76.7 \%)$ & 37 (63.8\%) & 0.126 \\
\hline LVEF, \% & $28.4 \pm 9.1$ & $25.9 \pm 10.6$ & 0.155 \\
\hline \multicolumn{4}{|l|}{ Clinical variables } \\
\hline \multicolumn{4}{|l|}{ NYHA classification } \\
\hline ॥ & I5 (25.4\%) & $16(28.1 \%)$ & 0.862 \\
\hline III & $36(61 \%)$ & 35 (6I.4\%) & \\
\hline IV & $8(13.6 \%)$ & $6(10.5 \%)$ & \\
\hline \multicolumn{4}{|l|}{ Laboratory tests } \\
\hline $\begin{array}{l}\text { NT-pro-BNP (ng/L), } \\
\text { median (IQR) }\end{array}$ & $\begin{array}{l}824 \\
(268 ; 1,596)\end{array}$ & $\begin{array}{l}1,862 \\
(553 ; 18,224)\end{array}$ & 0.268 \\
\hline \multicolumn{4}{|l|}{ Questionnaires } \\
\hline MLHFQ_TOT & $46.3 \pm 25.1$ & $47.2 \pm 20.6$ & 0.840 \\
\hline MLHFQ_PHY & $23.8 \pm 11.9$ & $23.7 \pm 10.5$ & 0.899 \\
\hline MLHFQ_EMO & $8.1 \pm 7.1$ & $8.4 \pm 6$ & 0.553 \\
\hline HADs_D & $5.8 \pm 4.3$ & $5.8 \pm 3.9$ & 0.886 \\
\hline HADs_A & $6 \pm 4.6$ & $5.1 \pm 4.2$ & 0.358 \\
\hline
\end{tabular}

Note: Values are presented as mean \pm SD.

Abbreviations: ICT-guided DMS, information-and-communication-technologyguided disease management system; TM+ICT-guided DMS, telemonitoring with ICT-guided disease management system; LVEF, left ventricular ejection fraction; NYHA, New York Heart Association; NT-pro-BNP, N-terminal pro-brain natriuretic peptide; IQR, interquartile range; MLHFQ_TOT, Minnesota Living with Heart Failure Questionnaire - total scale; MLHFQ_PHY, MLHFQ - physical subscale; MLHFQ_EMO, MLHFQ - emotional subscale; HADs_D, Hospital Anxiety and Depression scale - Depression subscale; HADs_A, HADs - Anxiety subscale.

\section{The impact of TM on self-care}

The baseline level of self-care assessed by the total score of EHFScBs was significantly lower (meaning better self-care) in the ICT-guided-DMS group $(n=60,19.2 \pm 7.7)$, compared to the TM+ICT-guided-DMS group $(\mathrm{n}=58,16.3 \pm 7.5, p=0.023)$. In the course of the study, self-care behavior significantly improved in the ICT-guided-DMS group (from 19.2 \pm 7.7 to $16.8 \pm 6.9, p<0.01)$ but not in the TM+ICT-guided-DMS group (from 16.3 \pm 7.5 to $15.4 \pm 6.7, p=0.77$ ) (Figure 1). At the end of the study, no statistically significant difference in the self-care behavior score between the groups was found.

\section{Dynamics of self-care throughout the study}

Pooling the data of all patients in the study, we found that the level of self-care behavior remained stable in only $12 \%$ of study patients, whereas it improved in $49 \%$ and worsened in $39 \%$ of all study participants. Due to the good initial selfcare (15.57 \pm 7.8$)$ undertaken by those with an unchanged EHFScBs score, these patients were combined with the subgroup of patients with improved self-care for further analysis. The distribution of changes in self-care between 


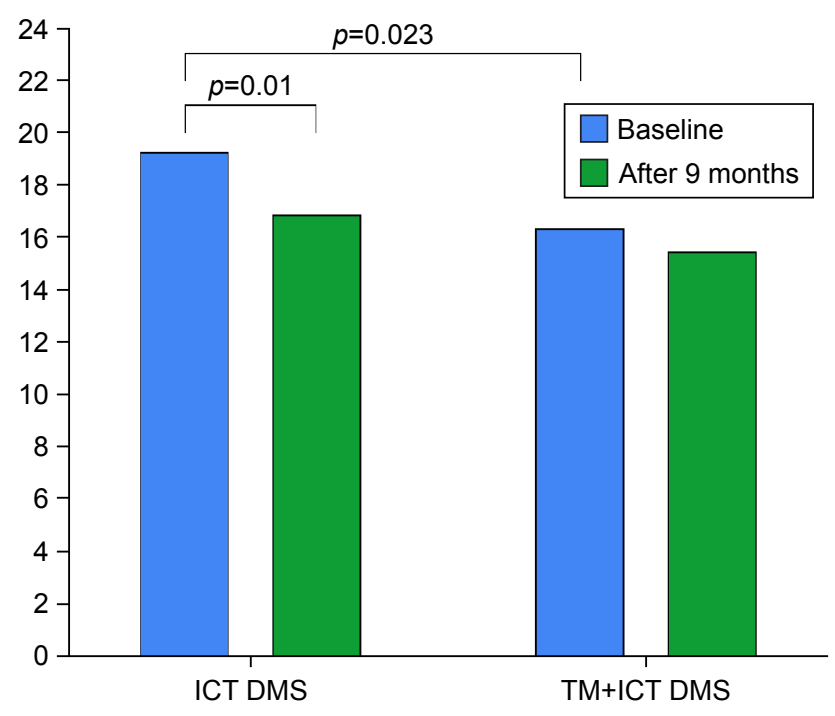

Figure I EHFScBs scores of the two groups at baseline and at 9 months. Abbreviations: DMS, disease management system; EHFScBs, European Heart Failure Self-care Behaviour scale; ICT, information and communication technology; TM, telemonitoring.

the two study groups ( $p=0.210$ between the groups) and in the whole study group is presented in Figure 2.

\section{Factors contributing to the worsening or improvement of self-care}

A higher baseline score of the physical subscale of the MLHFQ (OR 1.76, 95\% CI 1.143-2.712, per 10 points, $p<0.05$ ),

\section{EHFScBS}


Figure 2 Dynamics of self-care (improved, did not change, and worsened) among the groups and the whole study group.

Abbreviations: DMS, disease management system; EHFScBs, European Heart Failure Self-care Behaviour scale; ICT, information and communication technology; TM, telemonitoring. a lower LVEF (OR 0.75, 95\% CI 0.587-0.959, per 5\%, $p<0.05$ ), and a lower NYHA class (OR 0.251, 95\% CI $0.089-0.711$, III vs II class, $p<0.05$ ) were significantly associated with decreasing self-care behavior from baseline to the end of study (Table 2). Each 10-point increase of MLHFQ physical subscale was associated with a $76 \%$ increase in the chance of worsening self-care. Each drop of LVEF by $5 \%$ was related with $25 \%$ worsening of self-care. Patients in NYHA II had a 75\% higher chance of worsening self-care in comparison with NYHA III patients (Figure 3).

The only variable significantly related to a subsequent improvement in self-care was baseline LVEF: OR 1.25, 95\% CI 1.02-1.53, $p<0.05$. An increase in LVEF by $5 \%$ was associated with a $25 \%$ improvement in self-care.

\section{The characteristics of patients who showed the most improvement in HF self-care during the study}

Regression tree analysis showed that the greatest self-care improvement was observed in patients who had the lowest initial level of self-care, identified as an EHFScBs score $>28$. Another group with substantial self-care improvement was characterized by medium EHFScBs score (from 17 to 28) and concomitant HADs_D $<8$. Patients did not improve self-care in case of good initial self-care behavior (EHFScBs score $\leq 17)$ and self-care even worsened if it was combined with LVEF $\leq 28 \%$ (Figure 4). Neither TM nor NT-pro-BNP was disclosed as an important factor exerting effect on selfcare dynamics.

Depression (HADs_D >8) was additionally investigated in separate groups with different initial levels of self-care behavior. Depression was significantly related to HF selfcare deterioration not in the whole study group but in the subgroup with a medium range of EHFScBs scores (from 17 to 28), increasing the chances of self-care worsening by 10.22 times (95\% CI 1.81-57.69) (Table 3).

\section{Discussion}

Self-care is an important PRO for HF patients, which might be affected by disease management and/or TM. ${ }^{28}$ The present analysis of IN TOUCH data adds new findings and a new perspective to the investigations of self-care dynamics. The obtained results may stimulate novel design of interventions to improve self-care in HF patients. We found that self-care was not steady over time but remarkably changed, not always in the expected direction. We found that a high self-care score at one time point does not guarantee the same high self-care at a later point in time, implicating that an attention to self-care 
Table 2 Factors contributing to the worsening of self-care

\begin{tabular}{|c|c|c|c|c|c|c|c|c|}
\hline \multirow[t]{3}{*}{ Variables } & \multicolumn{4}{|c|}{ Univariate analysis } & \multicolumn{4}{|c|}{ Multivariate analysis } \\
\hline & \multirow[t]{2}{*}{ OR } & \multicolumn{2}{|c|}{ 95\% Cl for OR } & \multirow[t]{2}{*}{ p-value } & \multirow[t]{2}{*}{ OR } & \multicolumn{2}{|c|}{ 95\% Cl for OR } & \multirow[t]{2}{*}{$p$-value } \\
\hline & & Lower & Upper & & & Lower & Upper & \\
\hline NYHA & - & - & - & 0.136 & - & - & - & 0.033 \\
\hline Class II (reference) & - & - & - & - & - & - & - & - \\
\hline Class III & 0.421 & 0.177 & 1.000 & 0.050 & 0.251 & 0.089 & 0.711 & 0.009 \\
\hline Class IV & 0.703 & 0.197 & 2.507 & 0.587 & 0.362 & 0.077 & 1.691 & 0.196 \\
\hline NT-pro-BNP per I,000 ng/L & 1.002 & 0.990 & 1.014 & 0.730 & - & - & - & - \\
\hline MLHFQ_TOT per 10 points & 1.183 & 0.999 & 1.402 & 0.052 & - & - & - & - \\
\hline MLHFQ_PHY per 10 points & 1.450 & 1.013 & 2.074 & 0.042 & 1.760 & 1.143 & 2.712 & 0.010 \\
\hline MLHFQ_EMO per 10 points & 1.792 & 1.006 & 3.190 & 0.048 & - & - & - & - \\
\hline Age per I year & 0.984 & 0.953 & 1.017 & 0.337 & - & - & - & - \\
\hline LVEF per $5 \%$ & 0.783 & 0.630 & 0.972 & 0.027 & 0.750 & 0.587 & 0.959 & 0.022 \\
\hline Gender (male) & 0.672 & 0.301 & 1.499 & 0.331 & - & - & - & - \\
\hline +TM group & 1.879 & 0.888 & 3.978 & 0.099 & - & - & - & - \\
\hline HADs_A per I point & 0.968 & 0.888 & 1.055 & 0.460 & - & - & - & - \\
\hline HADs_D per I point & 1.093 & 0.997 & 1.199 & 0.059 & - & - & - & - \\
\hline
\end{tabular}

Note: Bold values represent significant differences.

Abbreviations: HADs_A, Hospital Anxiety and Depression scale - Anxiety subscale; HADs_D, HADs - Depression subscale.; LVEF, left ventricular ejection fraction; MLHFQ_EMO, MLHFQ - emotional subscale; MLHFQ_PHY, MLHFQ - physical subscale; MLHFQ_TOT, Minnesota Living with Heart Failure Questionnaire - total scale; NT-pro-BNP, N-terminal pro-brain natriuretic peptide; NYHA, New York Heart Association; TM group, telemonitoring group

in patient counseling during different phases of the HF disease trajectory is relevant. Furthermore, a poorer physical aspect of quality of life, worse ventricular function, and less severe symptoms (assessed as NYHA II vs NYHA III) were linked to decline in self-care during the study. The data confirm the significant relationship between self-care and functional status, presence of depression, and left ventricular function, the latter as a marker of disease severity. ${ }^{7}$
In TM programs, the effect on PROs, such as HF selfcare, is less commonly reported than are outcomes such as mortality, morbidity, and quality of life. ${ }^{17,29}$ Controversial results have been published until now regarding the potential of TM to improve self-care. ${ }^{17,18,29,30}$ Seto et al ${ }^{31}$ suggested that a TM system could improve HF patients' self-care by increasing the patients' awareness and knowledge regarding their HF condition, reducing anxiety, empowering, and motivating. ${ }^{31}$

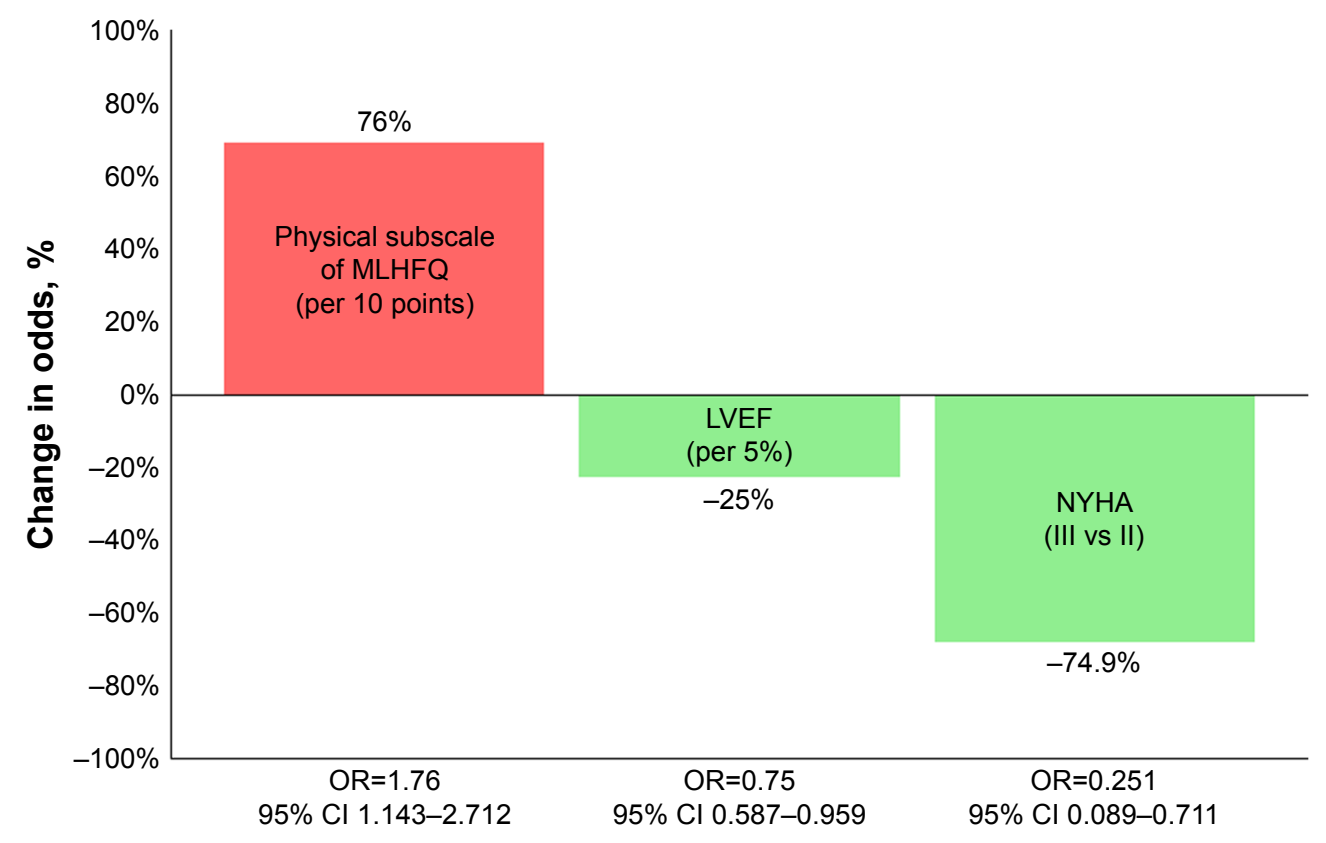

Figure 3 Factors associated with worsening of self-care.

Abbreviations: LVEF, left ventricular ejection fraction; MLHFQ, Minnesota Living with Heart Failure Questionnaire; NYHA, New York Heart Association. 


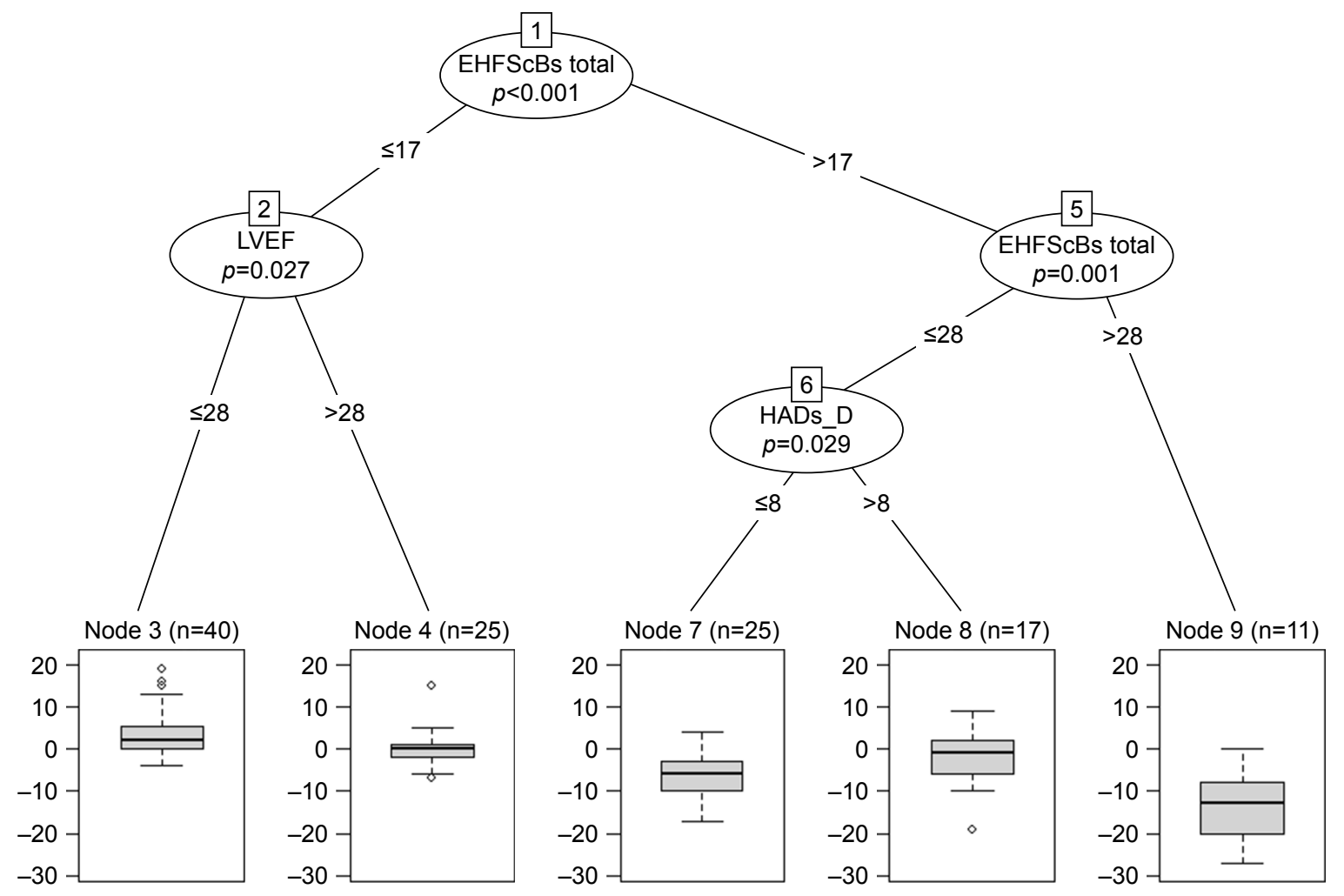

Figure 4 Regression tree showing the variables influencing changes in self-care.

Notes: Inputs in the tree model $(\mathrm{n}=1 / 8)$ were the following: intervention group, gender, LVEF, smoking, age, MLHFQ - total scale/physical subscale/emotional subscale, EHFScBs, HADs - Total scale, HADs_D, HADs_A, NYHA class, and NT-pro-BNP. Box plots show the distribution of the change in EHFScBs total score between baseline and 9 months. Central lines represents medians, boxes represent the 25th and 75th percentiles, and whiskers extend to the lowest data point within I.5 IQR of the lower quartile and the highest data point within I.5 IQR of the upper quartile. Dots represent extreme values.

Abbreviations: EHFScBs_TOT, European Heart Failure Self-care Behaviour scale - total score; HADs A, Hospital Anxiety and Depression scale - Anxiety subscale: HADs_D, Hospital Anxiety and Depression scale - Depression subscale; IQR, interquartile range; LVEF, left ventricular ejection fraction; MLHFQ, Minnesota Living with HF Questionnaire; NT-pro-BNP, N-terminal pro-brain natriuretic peptide; NYHA, New York Heart Association.

An improvement in self-care was demonstrated in five studies in a review by Radhakrishnon and Jacelon, ${ }^{32}$ but in five other studies, no difference in self-care between the intervention and control groups was observed. Similarly, a recent Cochrane systematic review ${ }^{17}$ demonstrated an improvement in self-care behavior scores and adherence to medications in six trials of structured telephone support and TM, in parallel with two neutral studies. Both reviews, however, mention the use of diverse evaluation tools and quality in the studies. Therefore, the questions remain whether TM indeed improves self-care, helps patients to understand the disease better, and stimulates the progress in self-care abilities. One even might

Table 3 Association of depression with worsening of self-care

\begin{tabular}{lllll}
\hline Variable & \multicolumn{3}{l}{ Univariate analysis } \\
\cline { 2 - 4 } & OR & \multicolumn{2}{l}{$\mathbf{9 5 \%} \mathbf{C l}$ for $\mathbf{O R}$} & p-value \\
\cline { 3 - 4 } & & Lower & Upper & \\
\hline EHFScBs_total $>28$ & 1.876 & 0.82 & 4.25 & 0.137 \\
EHFScBs $\leq 17$ & $1.37 \mathrm{I}$ & 0.40 & 4.76 & 0.619 \\
$\mathrm{I7}<$ EHFScBs $\leq 28$ & 10.22 & $\mathrm{I} .8 \mathrm{I} \mathrm{I}$ & $57.69 \mathrm{I}$ & 0.008 \\
\hline
\end{tabular}

Abbreviation: EHFScBs, The European Heart Failure Self-care Behaviour Scale. speculate that automated procedures using TM, combined with the perception of sharing of personal data, and external supervision can negatively influence self-responsibility and the patient's own mobilization in self-management.

The absence of a positive impact of TM on self-care in the IN TOUCH study could be explained by several reasons. First, the study comprised two intervention groups without a control group, with the consequence that all study patients regardless of group allocation received enhanced supervision of health care providers assisted by an ICT-guided DMS. This care program, implemented for both groups, maybe a potent management tool itself, leaving little room for the additional effect of automatic transmission of weight, blood pressure, and ECG data. Second, despite the use of a computer-generated randomization scheme, baseline self-care was significantly different between the two groups, with the TM+ICT-guidedDMS group patients reporting better self-care skills during randomization. Over the course of the study, the TM+ICTguided-DMS group did not improve, whereas self-care behavior in the ICT-guided-DMS group improved significantly. 
In contrast to the majority of the published data, the changes in the dynamics of self-care in the IN TOUCH study population were significantly related to two subjective parameters of functional status: NYHA class and the physical subscale of MLHFQ. ${ }^{7,33}$ A poorer baseline self-reported physical condition was associated with worsening of self-care during the study. However, in contrast, we found that a better baseline functional status (NYHA II) was associated with more chances of worsening of self-care. A possible explanation for this counterintuitive finding might be that subjects in a better functional condition (NYHA II vs NYHA III) feel smaller disease burden and might be less motivated in coping with the chronic illness. This was previously described by Nieuwenhuis et al, ${ }^{34}$ who found that patients in NYHA II more often had low long-term compliance compared to those in NYHA class III or IV. The authors explained this finding by a lack of motivation to adhere to the recommendations in case of modest symptoms associated with HF. ${ }^{34}$

In parallel with previous findings, ${ }^{8}$ better results in improvement of self-care were achieved if patients did not suffer from depression. Depression and anxiety are common among HF patients and are related to a poorer quality of life, self-care, ${ }^{35}$ adverse prognosis, ${ }^{36,37}$ and inadequate efficiency of educational programs. ${ }^{1}$ Chang et al ${ }^{38}$ proposed that depressive symptoms had a negative influence on HF self-care by reducing self-care confidence. A number of TM trials also showed that interventions were more effective in the absence of depression. ${ }^{39,40}$

One of the most important determinants of change in self-care behavior was its initial level. Our results, derived using regression tree, may be useful in the patients' selection for interventions aiming to improve self-care. This innovative analysis automatically generated a patient profile, described by specific characteristics and thresholds, which may be likely to benefit from DMSs. Educative measures may be most effective in HF patients with low baseline self-care score and absence of depression. The scores of the European Heart Failure Self-care Behaviour scale and the Hospital Anxiety and Depression scale could be used as the selection criteria when choosing the HF patients most responsive to interventions. However, at the same time, we found that self-care is not stable in all patients, and it should be acknowledged that attention to self-care is necessary in all cases.

\section{Limitations}

The current analysis included only those IN TOUCH study patients who completed the self-care questionnaire at baseline and at the end of study. Some patients did not fill in the questionnaires probably because they were too sick at the moment of inclusion or at the end of the study, and some had passed away. However, according to the biomarker levels, there was no significant difference in the severity of the disease between the analyzed subgroup and the total study population. Although this study was not specifically designed to investigate self-care dynamics, this is one of the few papers that describes changes in self-care over time, and these data add qualitatively to the understanding that selfcare is more complex than clinicians sometimes believe. Self-care is not always performed as a result of reflection and rational decision-making, ${ }^{1}$ and this paper contributes to the indication of the need to regularly assess self-care as a significantly changing behavior.

\section{Conclusion}

Self-care demonstrated a dynamic pattern in the majority of study patients. TM did not have an impact on self-care in addition to the ICT-guided DMS. Poor physical aspect of quality of life, lower ejection fraction, and lower NYHA class were factors associated with worsening of self-care. The greatest improvement in self-care may be achieved in those patients who have low - or medium - initial self-care level, in the absence of depression.

\section{Data availability}

The data sets used and/or analyzed during the current study are available from the corresponding author on reasonable request.

\section{Acknowledgments}

The IN TOUCH study was funded by the Dutch Ministry of Health, Department of Pharmaceutical Affairs and Medical Technology (GMT). The data analysis included in this article was supported by NordForsk's “Nordic Programme on Health and Welfare" (project number 76015). We thank all the participating Dutch heart failure clinics participating in the "IN TOUCH" study for their involvement, work, and cooperation.

\section{Author contributions}

All authors participated in substantial contributions to conception and design, data acquisition, or data analysis and interpretation; drafting the article or critically revising it for important intellectual content; final approval of the version to be published; and agree to be accountable for all aspects of the work in ensuring that questions related to the accuracy 
or integrity of the work are appropriately investigated and resolved.

\section{Disclosure}

JC received honorary fees from Novartis, Orivas, Grindex, and Amgen. The authors report no other conflicts of interest in this work.

\section{References}

1. Riegel B, Jaarsma T, Strömberg A. A middle-range theory of self-care of chronic illness. ANS Adv Nurs Sci. 2012;35(3):194-204.

2. van der Wal MHL, van Veldhuisen DJ, Veeger NJ, Rutten FH, Jaarsma T. Compliance with nonpharmacological recommendations and outcome in heart failure patients. Eur Heart J. 2010;31(12):1486-1493.

3. Ditewig JB, Blok H, Havers J, van Veenendaal H. Effectiveness of selfmanagement interventions on mortality, hospital readmissions, chronic heart failure hospitalization rate and quality of life in patients with chronic heart failure: a systematic review. Patient Educ Couns. 2010; 78(3):297-315.

4. Jonkman $\mathrm{NH}$, Westland $\mathrm{H}$, Groenwold RH, et al. What are effective program characteristics of self-management interventions in patients with heart failure? An individual patient data meta-analysis. J Card Fail. 2016;22(11):861-871.

5. Jonkman NH, Westland H, Groenwold RH, et al. Do self-management interventions work in patients with heart failure? An individual patient data meta-analysis. Circulation. 2016;133(12):1189-1198.

6. Jaarsma T, Strömberg A, Ben Gal T, et al. Comparison of self-care behaviors of heart failure patients in 15 countries worldwide. Patient Educ Couns. 2013;92:114-120.

7. Sedlar N, Lainscak M, Mårtensson J, Strömberg A. Factors related to self-care behaviours in heart failure: A systematic review of European Heart Failure Self-Care Behaviour Scale studies. Eur J Cardiovasc Nurs. 2017;16(4):272-282.

8. Dickson VV, Tkacs N, Riegel BA. Cognitive influences on selfcare decision making in persons with heart failure. Am Heart J. 2007;154(3): 424-431.

9. Alosco ML, Spitznagel MB, Cohen R, et al. Cognitive impairment is independently associated with reduced instrumental activities of daily living in persons with heart failure. J Cardiovasc Nurs. 2012;27(1):44-50.

10. Harkness K, Heckman GA, Akhtar-Danesh N, Demers C, Gunn E, McKelvie RS. Cognitive function and self-care management in older patients with heart failure. Eur J Cardiovasc Nurs. 2014;13(3):277-284.

11. Hajduk AM, Lemon SC, McManus DD, et al. Cognitive impairment and self-care in heart failure. Clin Epidemiol. 2013;5:407-416.

12. Ausili D, Rebora P, Di Mauro S, et al. Clinical and socio-demographic determinants of self-care behaviour in patients with heart failure and diabetes mellitus: A multicentre cross-sectional study. Int J Nurs Stud. 2016;63:18-27.

13. Dekker RL, Lennie TA, Doering LV, Chung ML, Wu JR, Moser DK. Coexisting anxiety and depressive symptoms in patients with heart failure. Eur J Cardiovasc Nurs. 2014;13(2):168-176.

14. Chaudhry SI, Phillips CO, Stewart SS, et al. Telemonitoring for patients with chronic heart failure: a systematic review. J Card Fail. 2007;13(1): $56-62$.

15. Clark RA, Inglis SC, McAlister FA, Cleland JG, Stewart S. Telemonitoring or structured telephone support programmes for patients with chronic heart failure: systematic review and meta-analysis. BMJ. 2007; 334(7600): 942.

16. Inglis SC, Clark RA, McAlister FA, Stewart S, Cleland JG. Which components of heart failure programmes are effective? A systematic review and meta-analysis of the outcomes of structured telephone support or telemonitoring as the primary component of chronic heart failure management in 8323 patients: abridged Cochrane review. Eur J Heart Fail. 2011;13(9):1028-1040.
17. Inglis SC, Clark RA, Dierckx R, Prieto-Merino D, Cleland JG. Structured telephone support or non-invasive telemonitoring for patients with heart failure. Heart. 2017;103(4):255-257.

18. Vuorinen AL, Leppänen J, Kaijanranta H, et al. Use of home telemonitoring to support multidisciplinary care of heart failure patients in Finland: randomized controlled trial. J Med Internet Res. 2014; 16(12): 282 .

19. Kraai I, de Vriesa A, Vermeulenb K, et al. The value of telemonitoring and ICT-guided disease management in heart failure: Results from the IN TOUCH study. Int J Med Inform. 2016;85(1):53-60.

20. de Vries AE, de Jong RM, van der Wal MH, Jaarsma T, van Dijk RB, Hillege HL. The value of INnovative ICT guided disease management combined with telemonitoring in OUtpatient clinics for Chronic Heart failure patients. Design and methodology of the IN TOUCH study: a multicenter randomised trial. BMC Health Serv Res. 2011; 11:167.

21. Jaarsma T, Arestedt KF, Martensson J, Dracup K, Stromberg A. The European Heart Failure Self-care Behaviour scale revised into a nineitem scale (EHFScB-9): a reliable and valid international instrument. Eur J Heart Fail. 2009;11(1):99-105.

22. Sokoreli I, de Vries JJ, Pauws SC, Steyerberg EW. Depression and anxiety as predictors of mortality among heart failure patients: systematic review and meta-analysis. Heart Fail Rev. 2016;21(1): 49-63.

23. Snaith RP, Zigmond A. The hospital anxiety and depression scale. Br Med J (Clin Res Ed). 1986;292(6516):344.

24. Garin O, Ferrer M, Pont A, et al. Evidence on the global measurement model of the Minnesota Living with Heart Failure Questionnaire. Qual Life Res. 2013;22(10):2675-2684.

25. Heo S, Maser D, Riegal B. Testing and published model of health-related quality of life in heart failure. J Card Fail. 2005;11:372-379.

26. Dickstein K, Cohen-Solal A, Filippatos G, et al. ESC guidelines for the diagnosis and treatment of acute and chronic heart failure 2008: the Task Force for the diagnosis and treatment of acute and chronic heart failure 2008 of the European Society of Cardiology. Developed in collaboration with the Heart Failure Association of the ESC (HFA) and endorsed by the European Society of Intensive Care Medicine (ESICM). Eur J Heart Fail. 2008;10(10):933-989.

27. Rokach LL. Decision forest: twenty years of research. Inform Fusion. 2016;27:111-125.

28. Riley JP, Gabe JPN, Cowie MR. Does telemonitoring in heart failure empower patients for self-care? A qualitative study. J Clin Nurs. 2013; 22(17-18):2444-2455.

29. Bashi N, Karunanithi M, Fatehi F, Ding H, Walters D. Remote monitoring of patients with heart failure: an overview of systematic reviews. J Med Internet Res. 2017;19(1):e18.

30. Boyne JJ, Vrijhoef HJ, Spreeuwenberg M, De Weerd G, Kragten J, Gorgels AP. Effects of tailored telemonitoring on heart failure patients' knowledge, self-care, self-efficacy and adherence: A randomized controlled trial. Eur J Cardiovasc Nurs. 2014;13(3):243-252.

31. Seto E, Leonard KJ, Cafazzo JA, Barnsley J, Masino C, Ross HJ. Perceptions and experiences of heart failure patients and clinicians on the use of mobile phone-based telemonitoring. J Med Internet Res. 2012;14(1):e25.

32. Radhakrishnan K, Jacelon C. Impact of telehealth on patient selfmanagement of heart failure: a review of literature. J Cardiovasc Nurs. 2012;27(1):33-43.

33. Riegel B, Moser DK, Anker SD, et al. State of the science: Promoting self-care in persons with heart failure: A scientific statement from the American Heart Association. Circulation. 2009;120:1141-1163.

34. Nieuwenhuis MM, Jaarsma T, van Veldhuisen DJ, Postmus D, van der Wal MH. Long-term compliance with nonpharmacologic treatment of patients with heart failure. Am J Cardiol. 2012;110(3): 392-397.

35. Kessing D, Denollet J, Widdershoven J, Kupper N. Psychological determinants of heart failure self-care: Systematic review and metaanalysis. Psychosom Med. 2016;78(4):412. 
36. Fan $\mathrm{H}$, Yu W, Zhang Q, et al. Depression after heart failure and risk of cardiovascular and all-cause mortality: A meta-analysis. Prev Med. 2014; $63: 36-42$.

37. Damen NL, Pelle AJ, Szabó BM, Pedersen SS. Symptoms of anxiety and cardiac hospitalizations at 12 months in patients with heart failure. J Gen Intern Med. 2012;27(3):345-350.

38. Chang LY, Wu SY, Chiang CE, Tsai PS. Depression and self-care maintenance in patients with heart failure: a moderated mediation model of self-care confidence and resilience. Eur J Cardiovasc Nurs. 2017; 16(5):435-443.
39. Prescher S, Deckwart O, Winkler S, Koehler K, Honold M, Koehler F. Telemedical care: feasibility and perception of the patients and physicians: a survey-based acceptance analysis of the telemedical interventional monitoring in heart failure (TIM-HF) trial. Eur J Prev Cardiol. 2013;20(2 suppl):18-24.

40. Koehler F, Winkler S, Schieber M, et al. Telemedicine in heart failure: Pre-specified and exploratory subgroup analyses from the TIM-HF trial. Int J Cardiol. 2012;161(3):143-150.

\section{Publish your work in this journal}

Patient Preference and Adherence is an international, peer-reviewed, open access journal that focuses on the growing importance of patient preference and adherence throughout the therapeutic continuum. Patient satisfaction, acceptability, quality of life, compliance, persistence and their role in developing new therapeutic modalities and compounds to optimize clinical outcomes for existing disease states are major areas of interest for the journal. This journal has been accepted for indexing on PubMed Central. The manuscript management system is completely online and includes a very quick and fair peer-review system, which is all easy to use. Visit http://www dovepress.com/testimonials.php to read real quotes from published authors.

Submit your manuscript here: http://www.dovepress.com/patient-preference-and-adherence-journal 